\title{
Distinguishing Propagation vs. Launch Physics of Astrophysical Jets and the Role of Experiments
}

\author{
Eric G. Blackman ${ }^{1}$ \\ 1. Dept. of Physics and Astronomy, Univ. of Rochester, Rochester, NY, 14627
}

\begin{abstract}
The absence of other viable momentum sources for collimated flows leads to the likelihood that magnetic fields play a fundamental role in jet launch and/or collimation in astrophysical jets. To best understand the physics of jets, it is useful to distinguish between the launch region where the jet is accelerated and the larger scales where the jet propagates as a collimated structure. Observations presently resolve jet propagation, but not the launch region. Simulations typically probe the launch and propagation regions separately, but not both together. Here, I identify some of the physics of jet launch vs. propagation and what laboratory jet experiments to date have probed. Reproducing an astrophysical jet in the lab is unrealistic, so maximizing the benefit of the experiments requires clarifying the astrophysical connection.
\end{abstract}

\section{Distinguishing Jet Launch vs. Jet Propagation Physics}

Jets in astrophysics emanate from accretion disk engines. The available jet mechanical luminosity is inversely proportional to the radius from the central engine, so the jet power is drawn from the inner most regions of the disk. Material must be accelerated to outflow speeds comparable to the escape speeds at the launch point. Radiation pressure is typically incapable of providing the directed momentum and instead various flavors of magnetic launch models remain the most promising. (see Livio 2004, Pudritz 2004 for reviews; also Lynden-Bell 2003).

Magnetic models take different forms. In steady-state "fling" models (e.g. Blandford \& Payne 1982), mass flux is sustained by centrifugal and toroidal magnetic pressure forces along the poloidal field. Explosive "spring" models (e.g. Wheeler, Meier, \& Wilson 2002; Matt et al. 2004; Moiseenko et al. 2006) also thrive on a gradient of magnetic field pressure, but are time dependent and do not require an initially imposed mass flux. Such "springs" may operate in gamma-ray bursts (GRB) and maybe supernovae. In both spring and fling models, the launch region is Poynting flux dominated but on scales $\lesssim 50 R_{\text {in }}$, (where $R_{\text {in }}$ is the scale of the inner engine) the jet becomes flow dominated. 
Springs and flings can be further distinguished from magnetic tower Poynting flux dominated outflow models (Lynden-Bell 2003; Uzdensky \& MacFadyen 2006); for the latter, Poynting flux domination remains even in the propagation region $\left(R \gtrsim 50 R_{i n}\right)$. Related models have been applied to GRB and active galactic nuclei (AGN) assuming the baryon loading is low. In the relativistic jets of AGN, microquasars, and GRB it is not certain how far in the propagation region the outflow remains $\mathrm{PF}$ dominated, In the non-relativistic jets of young stellar object (YSO), jets are baryon rich and likely flow dominated outside the launch region.

Presently, observations do not resolve the launch region at $R \lesssim 50 R_{\text {in }}$ for any source, although best indirect evidence for MHD launch perhaps comes from rotation of YSO jets $\lesssim 100$ AU scales (Coffey et al. 2004; Woitas et al. 2005). That $B$-fields are important to jet launching $\left(R \lesssim 50 R_{\text {in }}\right)$ is more widely agreed upon than the role of $B$ - fields in the asymptotic propagation region (despite the dearth of resolved observations of the former.) For example, if, by $\sim 50 R_{\text {in }}$, a magnetically collimated supersonic launch accelerates material to its asymptotic directed supersonic speed, then the tangent of the opening angle is just the inverse Mach number and the dynamical role of magnetic fields at larger radii may be inconsequential.

In all standard magnetic jet models, the magnetic field is dominant in a corona above the rotator, and the magnetic field has a large scale, at least compared to the scale of the turbulence in the rotator into which it is anchored. In recent magnetic tower models (Lynden-Bell 2003; Uzdensky \& McFadyen 2006), the tower has both signs of vertical magnetic flux since it is composed of loops anchored with both footpoints in the engine. Traditional MHD models which start with a large scale dipole field, produce a jet composed of one sign of magnetic flux and the return flux meanders at large distances, being dynamically unimportant. Instabilities in both geometries can disconnect blobs and produce knotty jets.

In short, the physics of the launch region (not yet resolved by astrophysical telescopes) involves such issues as: (1) Origin of magnetic fields, field buoyancy to coronae, magnetic helicity injection and relaxation into larger coronal structures, (2) physics of centrifugal + magnetic acceleration of material from small to super-Alfvénic speeds, or Poynting flux driven bursts of acceleration, (3) criteria for steady or bursty jets, and (4) assessment of the extent of Poynting flux domination.

The physics of the propagation region (resolved by astrophysical telescopes) involves such issues as: (1) Propagation, instability formation, and sustenance of collimation in as a function of internal vs. external density and strength of magnetic fields, (2) bow shocks, cocoon physics, particle acceleration, (3) effect of cooling on morphology, and (4) 
interaction with ambient media, stars, or cross-winds.

\section{Insights on Launch from the Sun and a Two-Stage Paradigm for Jet Fields}

Coronal holes and the solar wind provide an analogy to the more extreme jet launching from accretion disks. The launch region of the solar wind IS resolved. The coronal magnetic carpet (e.g. Schrijver \& Zwaan 2000) is composed of large scale "open" field lines as well as smaller scale "closed" loops. Both reverse every 11 years, so we know that the field is not a residue of flux freezing and must be produced by a dynamo.

There are three types of dynamos in astrophysics (e.g. Blackman \& Ji 2006): (1) Velocity driven small scale dynamos, for which magnetic energy amplification occurs without sustained large scale flux on spatial or temporal scales larger than the largest scale of the turbulence, (2) velocity driven large scale dynamos which can amplify field on scales larger than the largest turbulent scale, and (3) magnetically dominated large scale dynamos, also known as magnetic relaxation, whereby an already strong field, adjusts its geometry and such that any twists migrate to large scales. Both type (2) and type (3) involve magnetic helicity and an associated mean turbulent electromotive force aligned with the local mean magnetic field.

Type 1 and type 2 operate in the interior of a rotator, but some version of type 2, followed by a type 3 dynamo, provides the observable coronal field of the sun: First, a velocity driven helical dynamo amplifies fields of large enough scale that they buoyantly rise to the corona without shredding from turbulent diffusion. Once in the magnetically dominated corona, continued footpoint motions twist the field and inject magnetic helicity. In response, the loops incur instabilities which open up them or make them rise. Fields that power jets from disks may arise similarly.

The sun and disk are helicity injecting boundaries to their magnetically dominated corona, (analogous to spheromak helicity injection (Bellan 2000)). The type 2 dynamo occurs beneath the launch region and type 3 occurs in the launch region. Neither occurs in the propagation region.

\section{Insights on Propagation and Launch from Jet Experiments}

Astrophysical jet experiments are in their first incarnation, and presently involve non-relativistic jet motion. We cannot expect any experiment to reproduce any astrophysical source, but rather, address specific physics pieces. To gain insight on astrophysical problems, 
a careful assessment of how a given experiment specifically relates to the Sec. 1 distinction between jet formation and propagation is required.

\subsection{Insights from Coaxial Gun Helicity Injection Experiments: Launch}

Hsu \& Bellan (2002) employ a coaxial plasma gun composed of two coaxial electrodes linked by an axisymmetric vacuum magnetic field. This is analogous to an accretion disk with a dense set of poloidal magnetic loops, axisymmetrically distributed with zero initial toroidal field. At eight azimuthal locations, plasma is injected onto to the field lines while an electric potential is driven across the anchoring electrodes. An $\mathbf{E} \times \mathbf{B}$ toroidal rotation of the plasma results which then twists the poloidal field, amplifying a toroidal component. Equivalently, magnetic helicity is injected along the field.

Once the twist is injected and the toroidal field amplified, the loops rise and merge on the axis. (This is related to a type 3 dynamo, defined above.) A twisted unipolar core tower forms, rises, and remains collimated by hoop stress. The force free parameter $\alpha_{i n j} \equiv \mathbf{J} \cdot \mathbf{B} / B^{2}=I / \psi$ (where $I$ is the current from the imposed voltage across the electrodes and $\psi$ is the initial poloidal magnetic flux) measures the amount of twist injected. The measurements roughly agree with theoretical expectations of the Kruskal-Shafranov kink instability criterion. For $\alpha_{i n j} \leq 4 \pi / L$, where $L$ is the magnetic column length, the collimated structure is stable. When $\alpha_{i n j} \gtrsim 4 \pi / L$, the magnetic tower forms exhibits a kink instability but the structure stays connected. For $\alpha_{i n j}>>4 \pi / L$, the magnetic tower forms, a kink instability occurs, and a disconnected magnetic blob forms.

The experiments show that a kink instability need not immediately destroy jet collimation, even when disconnected blobs are produced. Real jets might be a series of ejected magnetically blobs, rather than a continuous flow. In astrophysics, pressure confinement may play an important role of collimating any magnetic tower.

The experiments probe jet formation in a plasma with $\beta \sim 0.02-0.1, T \sim 5-20 \mathrm{eV}$, fields of $\sim 1 \mathrm{kG}$, and number density $n=10^{14} / \mathrm{cm}^{3}$. The Alfvén Mach number $<1$ so this is a launch region experiment not a propagation region experiment. The value of $\alpha_{i n j}$ in real astrophysical system is determined by shear, resistivity, and coronal density.

\subsection{Insights from Pulsed Wire Array Experiments: Launch and Propagation}

For the pulsed power machine MAGPIE, Lebedev et al. (2005) set up radial array of tungsten wires arranged like spokes on a wheel (with a modest concavity) and applied a 
radial current. The current ablates the wires and produces a magnetic field around each. The mean magnetic field has a net toroidal component in the plane above the array and a net toroidal field of opposite sign below the array. The $\mathbf{J} \times \mathbf{B}$ force from the toroidal magnetic pressure accelerates material vertically. Toward the axis of the array, plasma is denser and an initial hydrodynamic precursor jet forms.

As the wires ablate and the magnetic force accelerates the plasma, a magnetically dominated cavity forms, Hoop stress collimates the denser plasma along the axis of the tower. The axis becomes a $\beta \sim 1$ plasma surrounded by $\beta<1$ toroidal magnetic field dominated cavity. Outside of the cavity is a $\beta>1$ ambient plasma supplied from the early stage of wire ablation. The dimensionless Reynolds and magnetic Reynolds numbers are larger than unity so the parameters are crudely OK for MHD an astro-comparison. Cooling is important as the cooling length is short compared to dynamical spatial scales. The experiment addresses principles of BOTH launch AND propagation physics.

The very narrowly collimated $\beta=1$ core jet has an internal Mach number of $\sim 4$. The surrounding large toroidal magnetic pressure driven cavity proceeds at Mach 10 with respect to the weakly magnetized ambient medium while the radial expansion is only Mach 3 so the cavity is collimated. In the experiments, these Mach numbers are already reached even when the tower height is only of order $\sim R_{i n}$, where $R_{i n}$ the array diameter $(\sim 4 m m$. $)$. That the vertical expansion is supersonic with respect to the ambient medium implies that the jet head has evolved from its formation region into its propagation region.

The structures produced in the experiments are analogous to pressure confined magnetic tower models (Lynden-Bell 2003; Uzdensky \& MacFadyen 2006), however there is very little polodial field, and the net toroidal field is produced from poloidal loops oriented perpendicular to the radial direction. A magnetically dominated tower encircling a $\beta=1$ highly collimated core may also apply to astrophysical jets.

At later stages of the evolution, the magnetic tower becomes kink unstable and a magnetic blob is ejected. But, as in Hsu \& Bellan (2002), here too the instability does not destroy the collimation of the tower. In this case, the ambient thermal pressure slows radial expansion. Blob formation again highlights the importance of time dependent dynamics, and that disconnected blobs may be the true nature of magnetized jets. Were more material available from the wires, the blob formation process in the experiment could repeat.

Though not the main focus of Lebedev et al. (2005), it is important to emphasize the precursor jet which precedes the magnetic cavity and results from the initially ablated plasma from the inner region of the wire array. This jet is hydrodynamic and collimated by radiative cooling. In fact, the analogue of this precursor jet is a close cousin to the the main 
focus of earlier conical wire array experiments of Lebedev et al. (2002) and Lebedev (2004). In these experiments, the conical array was more nearly cylindrical (concave at angles of 30 deg. with respect to the array axis rather than 80 deg.). Once the current is driven, this lower inclination implies an increased density on the axis of the jet compared to Lebedev (2005), thereby increasing cooling enough to break the flux freezing.

Lebedev et al. $(2002,2004)$ are thus supersonic hydrodynamic jet experiments. Given the discussion of Sec. 1, experiments for which the magnetic field is not important inside of jet are relevant at most to the propagation region, not the launch region. The particular

hydrodynamic jet experiments do show that that collimated supersonic launch may obviate the need for asymptotic magnetic collimation of a given jet when cooling is important. The collimation is enhanced when the wire material has a larger ion charge, enhancing radiative losses. This is consistent with a model of asymptotic protostellar jet collimation discussed in Tenorio-Tagle et al. (1988).

The Lebedev et al. (2002, 2004) experiments show Mach number $\geq 15$ jets. Jet deflection and shock propagation are studied in Lebedev et al. (2004) experiments, where an additional cross-wind is introduced into across the propagating jet flow. Generally, the hydrodynamic cooling-collimated jets seem to be relatively stable to non-axisymmetric perturbations.

\subsection{Insights from Laser Ablation Experiments: Propagation}

Another class of hydrodynamic jet experiments have been performed in laser inertial confinement facilities (Blue et al. 2005; Foster et al. 2005). These probe aspects of the jet propagation regime only. The experiments involve laser illumination of a thin metal disk such as titanium or aluminum. The thin target is placed flush against a washer about 6 times thicker, often of the same material. The lasers ablate the thin target disk and the ablated plasma is driven through the washer hole, exiting the hole in the form of a supersonic jet. The jet then propagates into a foam. A variety of features can be studied from the jet propagation into the foam using X-ray radiography and X-ray back-lighting.

Blue et al. (2005) report on experiments performed at NIF, They studied aspects of nozzle angle on jet structure by comparing axially symmetric (2-D) vs. titled (3-D) nozzles. The 3 -D case leads to an earlier transition to turbulence than in the 2-D case. Code testing of 2-D vs 3-D effects and the efficacy of the 3-D radiative HD code HYDRA (Marinak et al. 96) was confirmed, although the Reynolds numbers of the experiment are $R=10^{7}$ while only $R=10^{2}-10^{3}$ in the simulations. 
Similar experiments performed by Foster et al. (2005), on OMEGA obtain Mach

numbers as high as 5. The images are somewhat clearer than in Blue et al (2005). Turbulent flows, dense plasma jets, bow shock structures are seen. Modeling was done using 2-D hyrdo simulations with RAGE (Gittings 1992). These experiments probe a jet and foam density ratio of $\rho_{j} / \rho_{a} \sim 1$. This is intermediate between YSO jets which have $\rho_{j}>\rho_{a}$ vs. AGN jets which may have $\rho_{j}<\rho_{a}$. The latter however, are relativistic, and the experiments involve only non-relativistic flows.

\section{REFERENCES}

Bellan P.M., 2000, Spheromaks, (Imperial College Press, London)

Blackman E.G., and Ji. H, 2006, in press, MNRAS, astro-ph/0604221

Blandford, R. D., \& Payne, D. G. 1982, MNRAS, 199, 883

Blue, B. E., et al. 2005, Physical Review Letters, 94, 095005

Coffey, D., Bacciotti, F., Woitas, J., Ray, T. P., \& Eislöffel, J. 2004, ApJ, 604, 758

Foster, J. M., et al. 2005, ApJL, 634, L77

Hsu, S. C., \& Bellan, P. M. 2002, MNRAS, 334, 257

Lynden-Bell, D. 2003, MNRAS, 341, 1360

Lebedev et al. 2002, ApJ 563,113

Lebedev et al. 2004,ApJ 616, 988

Lebedev et al. 2005 MNRAS

Livio, M. 2004, Baltic Astronomy, 13, 273

Matt, S., Frank, A., \& Blackman, E. G. 2004, ASP Conf. Ser. 313: Asymmetrical Planetary Nebulae III: Winds, Structure and the Thunderbird, 449

Moiseenko, S. G, Bisnovatyi-Kogan, G. S., Ardeljan, N. V., 2006 submitted to MNRAS, astro-ph/0603789

Pudritz, R. E. 2004, Astrophys. \& Space Science, 292, 471 
Schrijver C.J. \& Zwaan C., 2000, Solar and Stellar Magnetic Activity, (Cambridge: Cambridge Univ. Press)

Tenorio-Tagle, G., Canto, J., \& Rozyczka, M. 1988, A\& A, 202, 256

Uzdensky, D. A., \& MacFadyen, A. I. 2006, submitted to ApJ, astro-ph/0602419

Wheeler, J. C., Meier, D. L., \& Wilson, J. R. 2002, ApJ, 568, 807

Woitas, J., Bacciotti, F., Ray, T. P., Marconi, A., Coffey, D., \& Eislöffel, J. 2005, A\&A, 432,149 